was 0.966 for the $125 \mathrm{mg}$ BID dose group and 1.014 for the 62.5 mg BID dose group (CI, 0.885-1.144). Side effects experienced by the $62.5 \mathrm{mg}$ BID group were, curiously, slightly higher than those of $125 \mathrm{mg}$ BID dose group, but these differences diminished when controlling for participant weight.

Conclusions.-A reduced dose of acetazolamide $62.5 \mathrm{mg}$ BID is as effective as the currently recommended dose of $125 \mathrm{mg}$ BID for the prevention of AMS. These results could influence future recommendations for climbers and trekkers with ascent profiles similar to those seen in our study.

\section{Sleep Characterization at High Altitude}

Nicholas Kanaan ${ }^{1}$; Caleb Phillips ${ }^{2}$; Becky Higbee ${ }^{3}$; David Pomeranz ${ }^{4}$; Michael Shaheen ${ }^{3}$; Kristin Fontes ${ }^{3}$; Patrick Cain $^{5}$; Sean Wentworth ${ }^{6}$; Carolyn Meyer ${ }^{7}$; Grant Lipman ${ }^{8}$

${ }^{1}$ University of Utah, Salt Lake City, UT, USA, ${ }^{2}$ University of Colorado at Boulder, Boulder, CO, USA, ${ }^{3}$ Stanford-Kaiser Emergency Medicine Residency, Stanford, CA, USA, ${ }^{4}$ LA County-USC Emergency Medicine Residency, Los Angeles, CA, USA, ${ }^{5}$ Midwestern University Emergency Medicine Residency, Glendale, AZ, USA, ${ }^{6}$ University of Arizona Emergency Medicine Residency, Tucson, AZ, USA, ${ }^{7}$ St. Luke's-Roosevelt Emergency Medicine Residency, New York City, NY, USA, ${ }^{8}$ Stanford University School of Medicine, Stanford, CA, USA

Introduction.-Poor sleep at high altitude is a welldescribed phenomenon and is associated with acute mountain sickness (AMS); however, large comprehensive studies characterizing sleep are lacking.

Objective.-This study aims to better understand factors contributing to the observed poor-quality sleep.

Methods.-We completed a descriptive analysis of the SLEEP-AID randomized controlled trial limited to the placebo $\operatorname{arm}(\mathrm{n}=104$ using resistance-free nasal strip, mean $\mathrm{LLQ}=1.86$, $17 \%$ AMS incidence) and analyzed 52 participants with complete data. Minute-by-minute sleep data and oxygen saturation were acquired with WatchPAT-200 sleep monitors on the first night of trekking to 4371 to $4530 \mathrm{~m}$ altitude in Nepal.

Results.-Participants were $70 \%$ male with an average age of 37 years (interquartile range [IQR] 27-48), and had mean 24-hour elevation gain of $448 \mathrm{~m}$ (IQR 380-550). Participants slept on average 477 minutes (IQR 442-517) with 11 awakening events (IQR 6-14). Their average sleep was 68\% light sleep (IQR 63-78), 11\% deep sleep (IQR 6-17), and 20\% rapid eye movement (REM) sleep (IQR 15-24). Average sleep latency was 22 minutes (IQR 19-26) with REM latency of 104 minutes (IQR 63-119). They experienced an average of 275 desaturation events (IQR 105-399), in which oxygen saturation dropped below $80 \%$, and spent $46 \%$ of the night below that threshold (IQR 11-84). All calculated sleep quality indices were profoundly abnormal: Respiratory Disturbance Index (mean 53, IQR 32-74), Apnea Hypopnea Index (mean 51, IQR 27-73), and Oxygen Desaturation Index (mean 37, IQR 14-54). All of these metrics (except \% REM sleep) were significantly worse in participants with AMS prior to sleep than in those who did not have AMS $(P<.05)$.

Conclusions. - This study more accurately describes altitude-related sleep metrics contributing to poor sleep. There were a large number of nocturnal desaturation and awakening events likely contributing to the hypobaric hypoxic insult of sleeping at high altitude and accounting for the profoundly abnormal sleep indices.

\section{Oral L-Tyrosine Supplementation Improved Core Temperature Maintenance to Whole-Body Cold Exposure in Older Adults}

James Lang ${ }^{1}$; Jahyun Kim ${ }^{2}$; Alex Krajek ${ }^{1}$; Joel Rand ${ }^{1}$

${ }^{1}$ Des Moines University, Des Moines, IA, USA, ${ }^{2}$ Iowa State

University, Ames, IA, USA

Introduction.-During cold exposure, an immediate and sustained increase in sympathetic nerve activity evokes vasoconstriction (VC) of cutaneous vessels to minimize heat loss and maintain body core temperature. In older adults, this reflex $\mathrm{VC}$ response is impaired, thereby increasing their susceptibility to excess heat loss and hypothermia. This may be explained in part by reduced bioavailability of the amino acid substrate, L-tyrosine, for catecholamine production. Thus, the thermoregulatory benefit of tyrosine supplementation in older adults is unknown.

Objective.-We hypothesize that oral L-tyrosine ingestion will augment the cutaneous $\mathrm{VC}$ response and thereby attenuate the decline in core temperature resulting from prolonged whole-body cooling in older adults.

Methods.-In a randomized, double-blind design, 8 older participants (aged $68 \pm 4$ years) ingested either $150 \mathrm{mg} / \mathrm{kg}$ of L-tyrosine or placebo before commencing 90 minutes of wholebody cooling to decrease skin temperature to $\sim 30^{\circ} \mathrm{C}$. Esophageal temperature (TES) and forearm laser Doppler flux was measured continuously throughout the protocol to provide an index of core temperature and skin blood flow, respectively. Cutaneous vascular conductance $(\mathrm{CVC})$ was calculated as $\mathrm{CVC}=$ laser Doppler flux / mean arterial pressure and expressed as a percent change from baseline (\% $\% \mathrm{CVC})$. The change in esophageal temperature ( $\Delta$ TES) was the difference in temperature at the end of cooling subtracted from baseline. Data were analyzed using 2-way (drug by cooling time) analysis of variance with repeated measures with Bonferroni post hoc analysis.

Results.-Oral tyrosine supplementation improved the reflex cutaneous VC response to cooling in older adults (placebo $=15.0 \pm 0.9$, tyrosine $=29.6 \pm 0.7 \% \Delta \mathrm{CVC}$; $P<.05)$. Additionally, tyrosine maintained body core temperature throughout cooling (placebo $=-0.31 \pm 0.04$, tyrosine $=-0.09 \pm 0.03 \Delta$ TES $P<.05)$.

Conclusions.-These results indicate that L-tyrosine supplementation may improve thermoregulatory function in response to acute cold exposure in an older population. 